\title{
Does mechanical stimulation really protect the architecture of trabecular bone? A simulation study
}

\author{
Manfred M. Maurer • Richard Weinkamer • \\ Ralph Müller • Davide Ruffoni
}

Received: 2 June 2014 / Accepted: 26 November 2014 / Published online: 14 December 2014

(C) Springer-Verlag Berlin Heidelberg 2014

\begin{abstract}
Although it is beyond doubt that mechanical stimulation is crucial to maintain bone mass, its role in preserving bone architecture is much less clear. Commonly, it is assumed that mechanics helps to conserve the trabecular network since an "accidental" thinning of a trabecula due to a resorption event would result in a local increase of load, thereby activating bone deposition there. However, considering that the thin trabecula is part of a network, it is not evident that load concentration happens locally on the weakened trabecula. The aim of this work was to clarify whether mechanical load has a protective role for preserving the trabecular network during remodeling. Trabecular bone is made dynamic by a remodeling algorithm, which results in a thickening/thinning of trabeculae with high/low strain energy density. Our simulations show that larger deviations from a regular cubic lattice result in a greater loss of trabeculae. Around lost trabeculae, the remaining trabeculae are on average thinner. More generally, thin trabeculae are more likely to have thin trabeculae in their neighborhood. The plausible consideration that a thin trabecula concentrates a higher amount of strain energy within itself is therefore only true when considering a single isolated trabecula. Mechano-regulated
\end{abstract}

M. M. Maurer · R. Müller · D. Ruffoni

Institute for Biomechanics, ETH Zurich, Zurich, Switzerland

\section{M. Maurer}

Institute of Mechanical Systems, ETH Zurich, Zurich, Switzerland

R. Weinkamer

Department of Biomaterials, Max Planck Institute of Colloids and Interfaces, Potsdam, Germany

D. Ruffoni $(\bowtie)$

Department of Aerospace and Mechanical Engineering,

University of Liege, Chemin des Chevreuils, 1, 4000, Liège, Belgium

e-mail: druffoni@ulg.ac.be remodeling within a network-like architecture leads to local concentrations of thin trabeculae.

Keywords Trabecular bone $\cdot$ Bone remodeling $\cdot$ Mechanical stimulation - Cellular solids · Mechano-regulation ·

Cubic lattice $\cdot$ Bone architecture

\section{Introduction}

Bone is organized either in the high-density type of cortical (or compact) bone or in the low-density type of trabecular (or cancellous) bone. In humans, trabecular bone is found in the vertebral bodies, in the long bones close to the joints and in the flat bones such as the ilium. Considering trabecular bone within the vertebrae, the bone volume fraction is roughly $15 \%$ for healthy individuals and decreases with age, in particular with osteoporosis, to values lower than $10 \%$ (Stauber and Müller 2006; Thomsen et al. 2013). Trabecular bone in the vertebrae is arranged in a network-like architecture, with the struts called trabeculae approximately forming a cubic lattice (Jensen et al. 1990; Yeh and Keaveny 1999), where the mean thickness of the trabeculae is about $140 \mu \mathrm{m}$ (Hildebrand et al. 1999). Considering the pivotal role of the trabecular architecture on the mechanical competence, especially in an osteoporotic scenario, the term architectural bone quality has been coined (Hernandez and Keaveny 2006; Seeman and Delmas 2006) to describe how the architecture (e.g., anisotropy and connectivity) influences the mechanical behavior. This research profits from computational studies on cellular solids, with trabecular bone idealized as a cellular structure consisting of a network of interconnected beams (Jensen et al. 1990; Lenthe et al. 2006). In trabecular bone, the mechanical role of various microstructural features of the trabecular network such as volume fraction, anisotropy, 
mean trabecular thickness, mean trabecular number and connectivity (Kabel et al. 1999; Yeh and Keaveny 1999; Guo and Kim 2002; Gibson 2005; Nazarian et al. 2006) as well as of single trabeculae like slenderness ratio and local trabecular orientation (Stauber et al. 2006; Liu et al. 2009; Easley et al. 2012) has been extensively detailed and interpreted according to the cellular solids framework. Moreover, the trabecular architecture being neither completely regular nor random (Fratzl and Weinkamer 2007), the role of disorder on both stiffness and strength is a particularly critical aspect. In cellular architectures, the localization of deformation and the subsequent failure are highly influenced by the presence of disorder and defects (Luxner et al. 2009a, b).

As a living material, trabecular bone has the intriguing property that its architecture can change and adapt to the mechanical needs. Biologically, this is possible due to the process of bone remodeling, where specialized cells resorb and deposit bone packets from and onto the surface (Parfitt 1994). This process is thought to be (at least partly) mechanically controlled, following the principle that bone is more likely deposited at sites in the network where local loading is high, and resorbed in locations of mechanical disuse (Schulte et al. 2013a). Computer simulations demonstrated that structural bone adaptation can be understood based on such a local mechano-regulation of bone (Huiskes et al. 2000; Tsubota et al. 2002; Dunlop et al. 2009; Hartmann et al. 2011; Schulte et al. 2013b). In the remodeling process, it is commonly believed that mechanical loading has a protective role not only in preserving bone mass (Frost 1987), but also in conserving the trabeculae (Smit and Burger 2000). This means that an "accidental" thinning of a trabecula would locally increase the mechanical strain in that location, which would be sensed by specialized cells (osteocytes) leading to the attraction of other cells (osteoblasts) for bone deposition. For a single and isolated trabecula, finite element (FE) calculations demonstrated this function of the mechanical stimuli and explained the natural succession of bone resorption and subsequent formation during remodeling (Smit and Burger 2000; Mulvihill et al. 2008). A recent FE study on a twodimensional regular honeycomb lattice including defects casts some doubt on this protective role of mechanical stimulation (Ruffoni et al. 2010). The study demonstrated that the reduction of thickness in a three-armed joint-i.e., the three struts meeting in a point of the network-of a regular honeycomb does not necessarily result in an increase in the local strain energy density (SED) in these struts. In contrast, the thinned struts, but also the struts in the closest environment, had low values of SED. Interpreting this result in terms of a mechano-regulated remodeling process implies that these already thin trabeculae would undergo further resorption with the risk of being eventually completely resorbed.

The aim of the current study was to analyze the connection between the trabecular architecture, in particular archi- tectural disorder, and the protective function of mechanical forces in a three-dimensional system which undergoes mechanically regulated remodeling. This protective function would be reflected in (i) that a loss of trabeculae is avoided, and therefore, the trabecular architecture is preserved. If the protection is not that rigorous, at least (ii) holes in the architecture formed due to the loss of trabeculae should be surrounded by rather thick trabeculae to avoid the local "weakness" spreading, which would undermine the mechanical performance of bone. To address this question, we employ an idealized model for trabecular bone with the following main features: Firstly, the model is three-dimensional with cylindrical trabeculae arranged in a cubic structure as observed, for example, in human vertebrae (Jensen et al. 1990). Secondly, the model considers a structural disorder that allows a variation in the thickness of the trabeculae and an architectural disorder that allows deviations from a perfect cubic lattice and its known mechanical singularities (Luxner et al. 2007). Thirdly, the trabecular architecture can evolve in time using a mechano-regulated remodeling algorithm. The mechanoregulation is characterized by a single parameter, a reference value for the strain energy density $\mathrm{SED}_{\text {ref }}$. If the local SED in a trabecula is above this reference value, the thickness is increased, where the increase is assumed to be linear with the excess of SED, but cannot exceed a maximum increase due to constraints given by bone biology. Likewise, resorption occurs for local values of the SED below SED $\mathrm{Def}_{\text {re }}$ Resorption can result in a complete loss of trabeculae, which cannot be restored once they are lost. In a simulation, this algorithm is iteratively applied for a total simulated time corresponding to roughly 50 years. To assess the role of mechanical stimulation for bone architecture, we analyze the overall bone volume, the frequency distributions of the thickness of the trabeculae and how these thicknesses are distributed spatially within the lattice. One additional focus is to study the local environment of either completely resorbed or unusually thin trabeculae, to investigate whether such mechanically weak points in the architecture are "protected" by thickened neighboring trabeculae.

\section{Methods}

\subsection{Idealized trabecular network}

In our model, trabecular bone was described as a threedimensional cubic lattice consisting of $20 \times 20 \times 20$ repeating unit cells with a lattice constant (corresponding to trabecular separation) of $1 \mathrm{~mm}$ (Fig. 1a). The volume of the virtual bone was therefore $8 \mathrm{~cm}^{3}$. The geometry of the individual trabeculae is simplified to cylindrical rods (Jensen et al. 1990; Guo and Kim 2002). To account for the natural variations in the thickness of real trabeculae, we assigned to each trabec- 


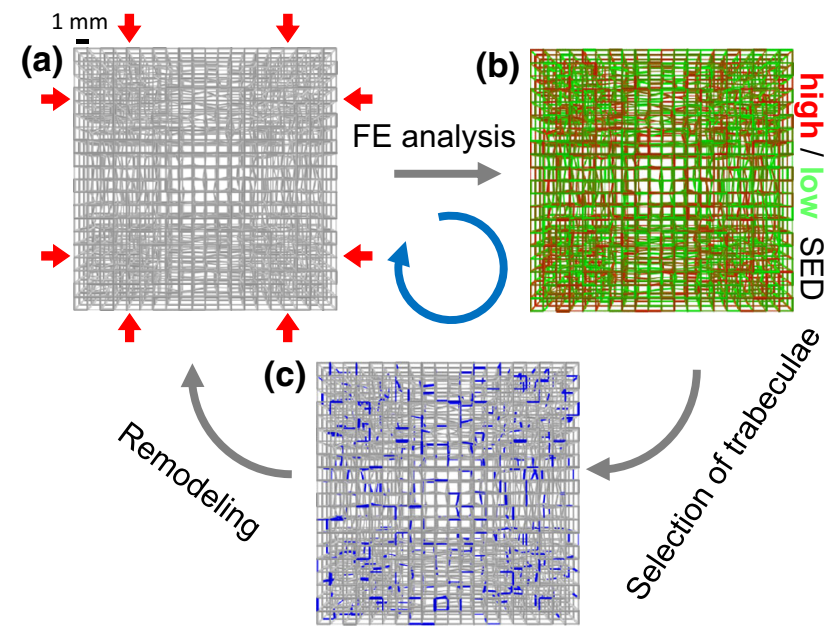

Fig. 1 Schematic illustration of the framework for trabecular bone remodeling. a The trabecular lattice is compressed by a hydrostatic force. $\mathbf{b}$ The strain energy density (SED) in each trabecula is computed by beam finite element (FE) analysis. $\mathbf{c}$ The trabeculae to be remodeled (blue) are randomly selected until the total remodeled surface reaches $10 \%$ of the total bone surface. Applying the remodeling rule (Eq. (1)) for each selected trabeculae, the new lattice configuration is obtained

ula a different thickness chosen from a Gaussian trabecular thickness distribution (mean $0.14 \mathrm{~mm}$, standard deviation $0.042 \mathrm{~mm}$ ), which is within the range of reported values for healthy human vertebrae (Hildebrand et al. 1999; Yeh and Keaveny 1999; Stauber and Müller 2006). Furthermore, as the trabecular network even in the vertebra does not have a regular cubic order (Jensen et al. 1990), some architectural disorder was introduced in the lattice by perturbing the location of joints (i.e., the intersection points of six trabeculae), excluding joints on the boundary of the cubic lattice. Specifically, the architectural disorder was attained by shifting each inner joint of the lattice, where the amount and direction of the shift was randomly selected from three-dimensional Gaussian distributions with mean equal zero and a standard deviation $\sigma$. The value of $\sigma$, given in units of the spacing of the regular lattice, characterizes the amount of architectural disorder. We studied lattices with a disorder of $0 \%$ (regular lattice), 10, 20, 30 and $40 \%$. For each value of disorder, five different initial architectures of the lattice to start the simulation with were generated to account for the variability in the architecture when disorder is introduced.

\subsection{Remodeling framework}

The iterative remodeling framework, visualized in Fig. 1, consisted of two main steps: first the step of mechanical assessment followed by the remodeling event. The trabecular lattice was compressed hydrostatically by a force of $680 \mathrm{~N}$ (uniformly distributed over all the surface nodes) corresponding to an apparent stress of 1.7 MPa (Dunlop et al. 2009), as reported for physiological loading of human vertebral bone
(Adams and Dolan 1995) (Fig. 1a). In order to prevent rigid body movements, one node at the corner of the lattice was fixed in all six degrees of freedom. The mechanical problem was solved with the commercial finite element analysis software ABAQUS/standard (Version 6.10-EF, Simulia, US). Each trabecula of the lattice was modeled with four beam elements with circular cross section (element B32 in ABAQUS). These are 3-node, second-order elements that account for axial, bending and shear deformation. The sufficient accuracy of such a meshing was demonstrated in previous studies (Luxner et al. 2005; Ruffoni et al. 2012a), where the simulated mechanical properties deviated less than $0.1 \%$ when using more than four beam elements per trabecula (Ruffoni et al. 2012a). Bone tissue was described as an isotropic linear elastic material with Young's modulus and Poisson's ratio of $10 \mathrm{GPa}$ and 0.3 , respectively, as used in earlier computational studies on trabecular bone (Ruffoni et al. 2012a, b) and in agreement with values reported in literature (Keaveny et al. 2001; Carretta et al. 2013). A typical mesh had around $10^{5}$ elements and required about $10 \mathrm{~min}$ to be solved under the assumption of small deformations on a normal PC (4 cores processor with $2.66 \mathrm{GHz}$ of frequency and $8 \mathrm{~GB}$ of primary memory). The main outcome of the FE analysis was the strain energy density (SED) stored in each trabecula (Fig. 1b).

In the second step of the algorithm, the trabeculae were then remodeled according to their local SED. In living human trabecular bone, not all trabeculae are remodeled at the same time. The percentage of the trabecular bone surface which is undergoing remodeling at a given time point is around $10 \%$ (Chavassieux et al. 1997) of the total trabecular surface as measured in standard bone histomorphometry (Parfitt et al. 1987). Here, we described remodeling by randomly selecting the trabeculae, which can undergo remodeling in an iteration of the simulation, until the total remodeled area was $10 \%$ of the current total surface area (Fig. 1c). How much of each selected trabecula was remodeled, was determined according to its local SED. The random selection process allowed each trabecula to have a chance to be remodeled, as remodeling does not occur exclusively at the locations with the highest or lowest strains (Schulte et al. 2013a). During a single remodeling event in trabecular bone, a roughly longitudinal and semi-cylindrical "trench" (Parfitt 1994; Jee 2001) is remodeled along the trabecula; in our model, this is reflected in a reduction or increase of the cross-sectional area and thus volume of the trabecula. To calculate in the model the change in the cross-sectional area $(\Delta A)$ of the selected trabecula as a function of the local loading, a deterministic remodeling rule was implemented, where $\Delta A$ was assumed linearly proportional to the local SED of the trabecula (Fig. 2a):

$$
\Delta A=\left\{\begin{array}{ll}
\frac{\Delta A_{\max }}{\mathrm{SED}} \mathrm{SED}-\Delta A_{\max }, & \mathrm{SED}<2 \mathrm{SED}_{\mathrm{ref}} \\
\Delta A_{\max }, & \mathrm{SED}>2 \mathrm{SED}_{\mathrm{ref}}
\end{array} .\right.
$$



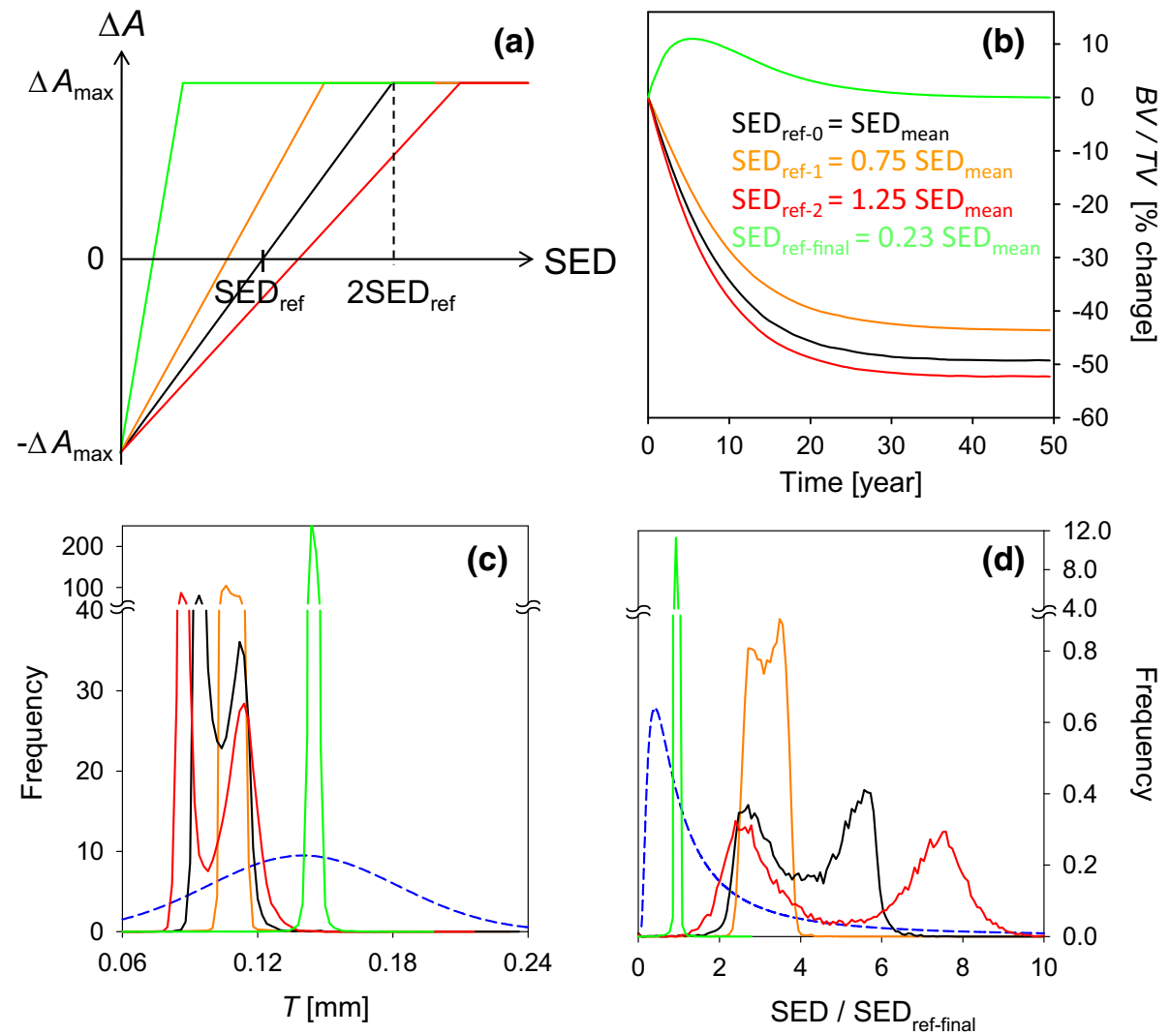

Fig. 2 a Deterministic remodeling rule (line in black) with the change in the cross-sectional area of a trabecula $(\Delta A)$ assumed linearly proportional to the local SED of the trabecula. The slope of the remodeling rule (i.e., the mechano-sensitivity of the remodeling process) increases by decreasing $\mathrm{SED}_{\text {ref }}$, which is the value of SED at which no remodeling takes place. The other different colored remodeling rules were used for simulations presented in plots (b)-(d). b Time evolution of the change in bone volume fraction (BV/TV) of the virtual bone for different values

The maximum bone resorption $\left(\Delta A_{\max }\right)$, occurring at zero SED, was estimated based on histomorphometric measures, assuming a resorption pit of a semi-cylindrical shape with a radius of $50 \mu \mathrm{m}$ (Parfitt 1994; Rusconi et al. 2012):

$\Delta A_{\max }=\frac{\pi(50 \mu \mathrm{m})^{2}}{2}=3925 \mu \mathrm{m}^{2}$.

Likewise, maximum bone formation was also bounded by the same value $\Delta A_{\max }$, corresponding to the amount of bone necessary to completely refill the biggest excavated pit. The SED value at which no remodeling takes place $(\Delta A=0)$ was referred to as reference SED (SED ref) (Fig. 2a). In line with our intention to keep the mechanical control of the remodeling process as simple as possible, $\mathrm{SED}_{\text {ref }}$ was the only free parameter in the proposed remodeling rule.

Due to a remodeling event, a trabecula having an initial thickness $T$ was either uniformly thinned or thickened by an amount $\Delta T$ equal to:

of SED ref. Final steady-state probability distributions of (c) trabecular thickness and (d) trabecular SED obtained with different $S_{E D}$ ref. The SED is normalized by the chosen value of reference SED, SED ref-final, corresponding to $23.05 \%$ of the initial mean SED in the lattice. The blue curve in (c) indicates the initial Gaussian probability distribution used for all simulations and in (d) the corresponding initial SED probability distribution at $0 \%$ disorder

$\Delta T=\sqrt{T^{2}+\frac{4 \Delta A}{\pi}}$.

As a result of an iteration step, a new lattice characterized by changes in the thickness of some of its trabeculae was generated, and it was used as input for the subsequent FE analysis. Simulations using this iterative algorithm were terminated after 100 iteration cycles corresponding to 50 years of remodeling. Within this time period, all the trabeculae in the lattice undergo remodeling at least once. The conversion of the iteration time to real time was performed knowing that one iteration corresponded to the period of a total remodeling cycle of about 6 months (Parfitt et al. 1987).

\section{Results}

3.1 Influence of the set point $\mathrm{SED}_{\text {ref }}$

Firstly, it was determined whether the system approaches a steady-state configuration, and if it does, this configuration 
was characterized. The simplicity of our approach has the advantage that the model includes only one "free"-i.e., not based on experimental data-parameter $\left(\mathrm{SED}_{\mathrm{ref}}\right)$ to define the remodeling rule (Fig. $2 a$ ). Figure $2 b$ shows the time evolution of the percentage changes of bone volume fraction $B V / T V$ (i.e., bone volume divided by tissue volume) for different values of $\mathrm{SED}_{\text {ref }}$ when starting the simulations from an architecturally fully ordered cubic lattice with a Gaussian thickness distribution (blue line, Fig. 2c and Appendix A). The value of $\mathrm{SED}_{\text {ref }}$ is given in units of the mean SED within the ordered cubic lattice after the initial hydrostatic compression, $\mathrm{SED}_{\text {mean }}$, and the corresponding SED distribution is plotted in Fig. 2d-blue curve (see also Appendix A). Choosing $\mathrm{SED}_{\text {ref }}=\mathrm{SED}_{\text {mean }}, B V / T V$ drops in time and attains an approximately constant value after 40 years with roughly half of the initial bone volume lost (Fig. 2b). The virtual bone is not mechano-sensitive enough, i.e., the value of $\mathrm{SED}_{\text {ref }}$ is too high, to preserve the bone volume. Preservation of the volume is achieved when choosing $\mathrm{SED}_{\text {ref }}=0.2305 \mathrm{SED}_{\text {mean }}$. In this case, the time evolution of $B V / T V$ exhibits a peak at about 8 years and then approaches its steady-state value from above (Fig. 2b). The corresponding trabecular thickness distributions and SED distributions obtained after 50 years for the different values of $S_{E D}$ ref are shown in Fig. $2 c$ and $d$, respectively. For low values of $\mathrm{SED}_{\text {ref }}$, the $\mathrm{SED}$ distribution is strongly peaked (Fig. 2d) as expected for an algorithm whose driving force is the homogenization of the SED. Also, the trabecular thickness distribution is peaked since the lattice is ordered, and consequently, the same value of the SED is obtained for a specific thickness of the trabecula. However, for high values of $\mathrm{SED}_{\text {ref }}$, a different behavior is observed with bimodal distributions for both SED and thickness. A detailed explanation of this observation will be provided later in the Discussion and Appendix B. All the subsequent simulations in this work are performed with $\mathrm{SED}_{\text {ref }}$ equal to $23.05 \%$ of the initial mean SED, which avoids large changes in the bone volume compared to the initial condition and hence allows to investigate the behavior of trabecular architecture independently from large variations in bone volume fraction.

\subsection{Role of architectural disorder on lattice structural evolution}

The next set of computer simulations tested the influence of architectural disorder on the dynamic behavior of the virtual bone lattice. For the initial conditions, an identical Gaussian trabecular thickness distribution was always used. As a consequence, the introduction of more disorder in the initial configuration of the lattice also leads to an increase in the initial value of the bone volume fraction, $B V / T V$. However, even in the case of highest disordered studied (i.e., $40 \%$ disorder), the increase of the initial $B V / T V$ was not larger

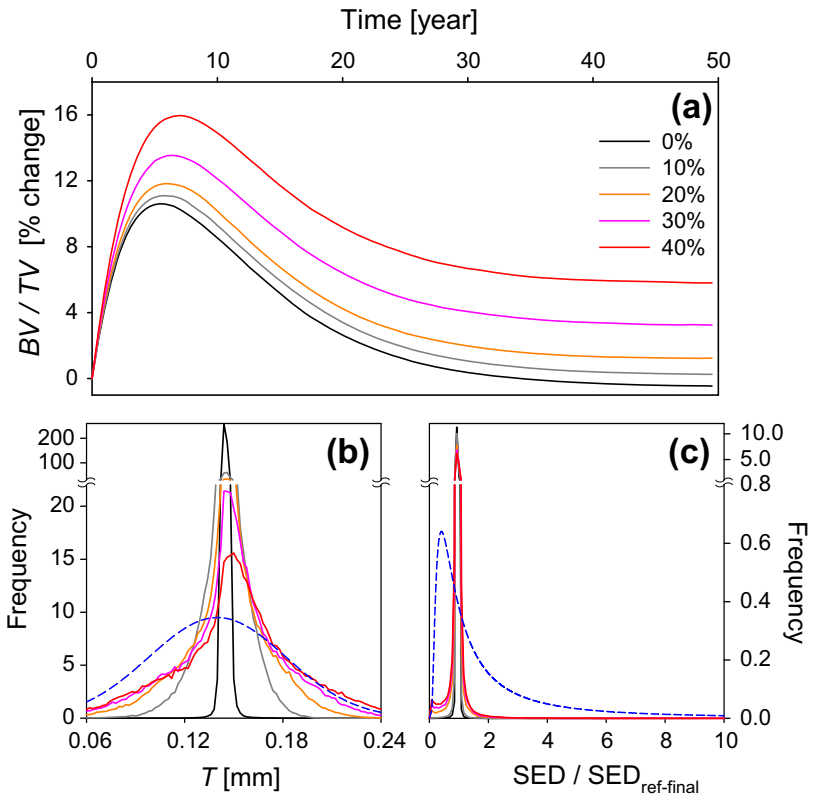

Fig. 3 a Time behavior of the change in bone volume fraction $(B V / T V)$ of the trabecular lattice for different levels of architectural disorder. Corresponding steady state $\mathbf{b}$ thickness and $\mathbf{c}$ SED distributions obtained by increasing the amount of architectural disorder from $0 \%$ (black) to $40 \%$ (red). The blue curves again indicate the frequency distributions at the beginning of the remodeling process

than $1.95 \%$. The reported changes of $B V / T V$ are normalized again to their initial value ( $0 \%$ at the start of all simulations) (Fig. 3a). The time evolution of $B V / T V$ for lattices with different amounts of architectural disorder shows a similar behavior as in the ordered lattice: After a peak in bone volume, which is attained before 10 years, the bone volume decreases again and approaches its steady-state value (Fig. 3a). The steady-state value of $B V / T V$ increases with disorder in a nonlinear way, since a change in disorder from 0 to $10 \%$ increases $B V / T V$ by only $0.25 \%$, while changing disorder from 30 to $40 \%$ results in a $2.4 \%$ increase (see also Fig. 4 ). For the largest architectural disorder of $40 \%$, the final value of $B V / T V$ is $6 \%$ larger than the initial volume. This $6 \%$ difference in $B V / T V$ is already almost present in the difference in peak heights between 0 and $40 \%$ disorder (Fig. 3a), so that the approach to steady state from the maximum value of $B V / T V$ is similar for all the studied lattices. That a steady-state configuration was attained at 50 years, when the remodeling simulations are stopped, is corroborated by looking at the corresponding SED distributions (Fig. 3c). Independent of the amount of disorder, the SED distributions display a sharp peak, centered on $\mathrm{SED}_{\mathrm{ref}}$, as expected from the mechanical regulation. However, to compensate for the architectural disorder, the trabecular thickness distributions are different for different amounts of disorder (Fig. 3b). While the peak position and the mean value hardly change with disorder (4 and $0.6 \%$, respectively, when changing the disorder from 10 


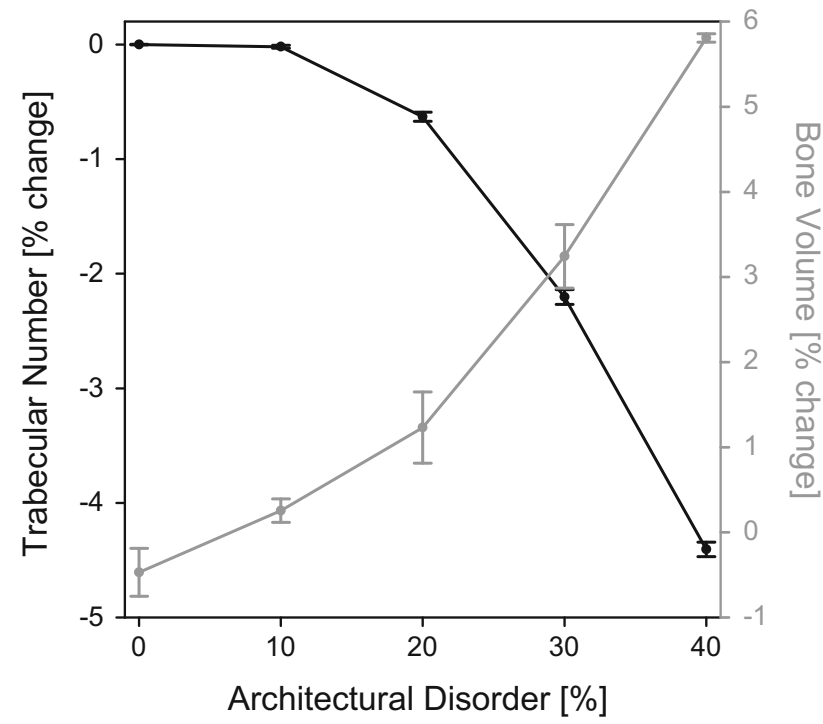

Fig. 4 Changes in the number of trabeculae in the lattice and in bone volume (normalized to the respective initial values before remodeling) for different amounts of architectural disorder. Data are shown as mean values \pm standard deviations over five simulations

to $40 \%$ ), the width of the distributions (measured as full width at half maximum) increases considerably by $24 \%$. Most remarkably, introducing architectural disorder in the lattice results in a loss of trabeculae through bone resorption. This effect is largest with a loss of about $4.4 \%$ of the total number of trabeculae for $40 \%$ disorder (Fig. 4). In this scenario, though, the thickening of the remaining trabeculae is so substantial that the bone volume, nevertheless, increases with disorder (Fig. 4).

\subsection{Spatial distributions of lost and thin trabeculae}

The observation of loss of trabeculae undergoing a mechanically controlled remodeling calls for a closer study of the environment of these "holes" in the lattice. Already the twodimensional cut through a lattice with $40 \%$ disorder at late times (Fig. 5a) gives the impression that holes are not surrounded by particularly thick trabeculae. On the contrary, in the marked regions of the plot, either more than one trabecula got lost or very thin trabeculae can be found. Figure $5 \mathrm{~b}$ presents a quantitative analysis of this impression, showing the distribution of the thickness of only those trabeculae which have in their nearest neighborhood (i.e., the 10 nearest-neighboring trabeculae in direct contact) either $0,1,2$ or 3 trabeculae which were completely resorbed. For a lattice having $40 \%$ disorder, the frequency distributions obtained in steady state are fairly symmetrical and can be well fitted by a Gaussian function $\left(R^{2}=0.98\right)$ to guide the eye. The distributions shift toward thinner trabeculae when the number of lost neighbors increased (Fig. 5b, red). The mean thick-
$T[\mathrm{~mm}]$

(a)
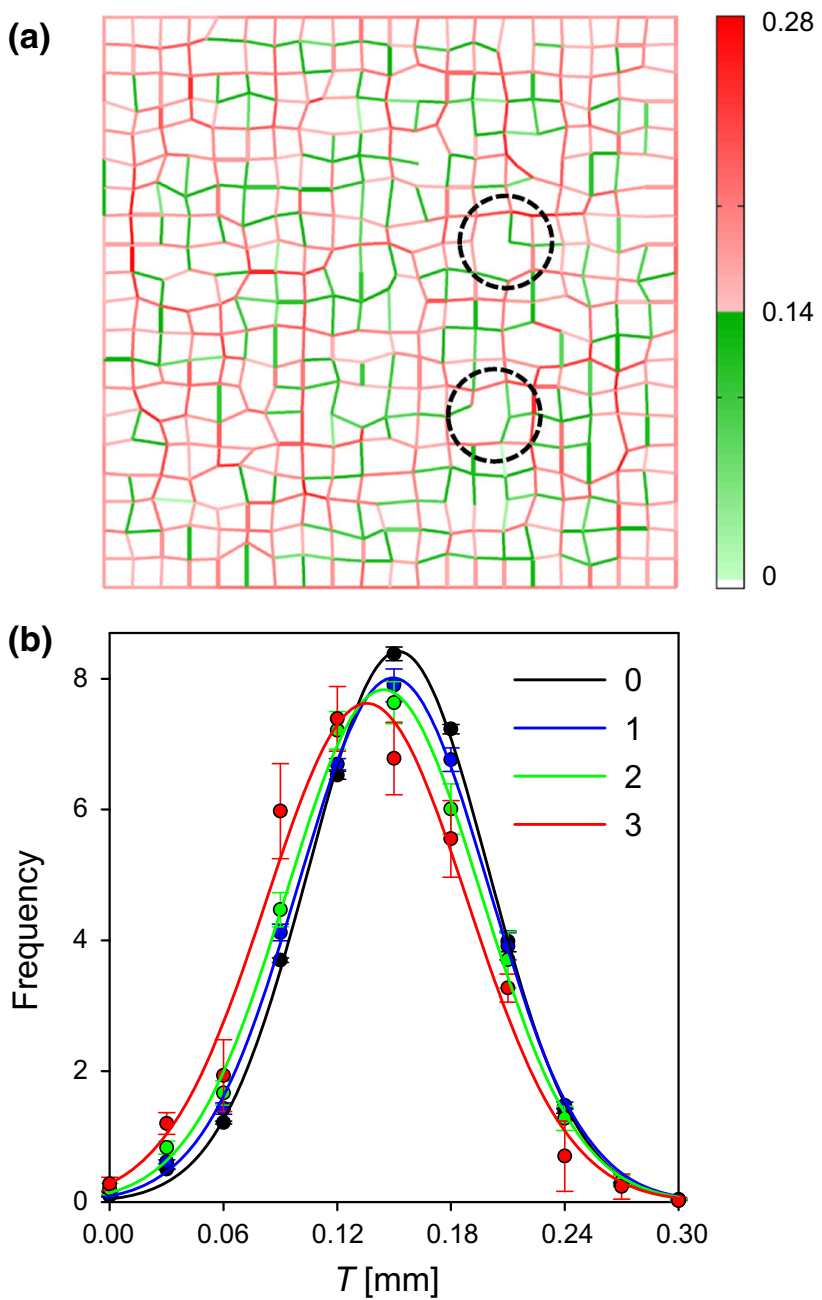

Fig. 5 a Two-dimensional cut through a trabecular lattice with $40 \%$ architectural disorder after 50 years of remodeling with the thickness of each trabecula color-coded: red indicates thick trabeculae and green thin ones. The black circles denote areas where the mechano-regulated remodeling process resulted in loss of trabeculae creating holes in the network: These holes are surrounded by relatively thin trabeculae. b Thickness distribution of the trabeculae which have in their nearest neighborhood (i.e., the 10 nearest-neighboring trabeculae in direct contact) either $0,1,2$ or 3 trabeculae which were completely resorbed during remodeling. These distributions could be well fitted $\left(R^{2}<0.98\right)$ by Gaussian functions. Data are shown as mean values \pm standard deviations over five simulations

ness of the trabeculae having in the neighborhood three holes decreases by about $10 \%$, and the heterogeneity (characterized again by the full width at half maximum) increases by approximately $8 \%$ compared to the local environment away from holes. Figure 6 provides a more general view on spatial correlations between thin and thick trabeculae, where the probability is plotted to find a trabecula of thickness $t_{2}$ in the nearest neighborhood of a trabecula having thickness $t_{1}$. The absence of short range correlations in the trabecular network in the initial configuration is demonstrated by the 


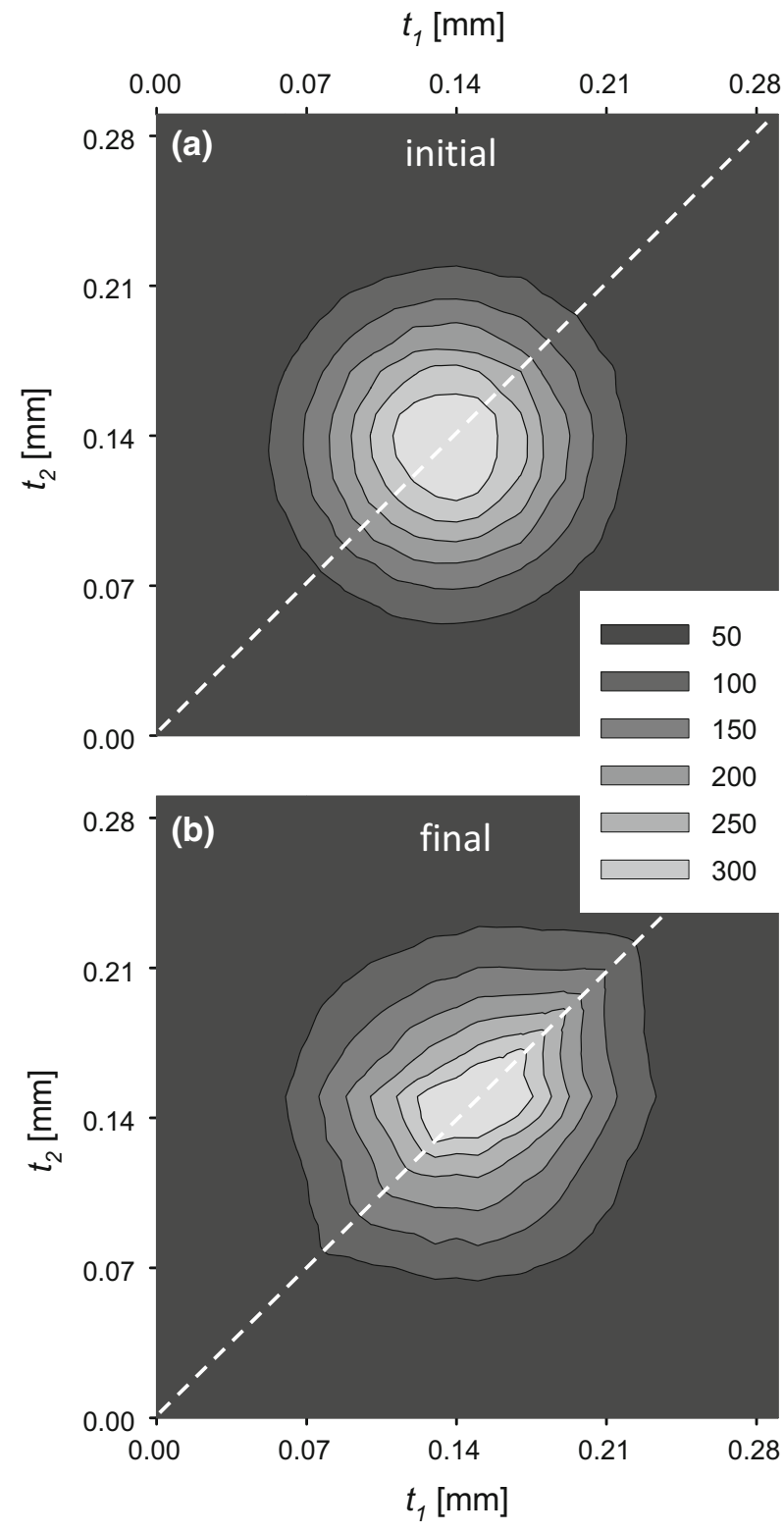

Fig. 6 Contour plots of the probability that a trabecula of thickness $t_{2}$ is found in the neighborhood (i.e., the 10 nearest-neighboring trabeculae in direct contact) of a trabecula having thickness $t_{1}$ for the virtual bone with $40 \%$ architectural disorder $\mathbf{a}$ at the beginning of remodeling and b after 50 years

circular contours (Fig. 6a), as a result of a random assignment for the trabecular thickness from a Gaussian distribution to obtain the initial configuration. With time, the plot develops an increasing ellipticity with a ratio of 1.3 between the major and minor axis in the steady state for $40 \%$ disorder (Fig. 6b). Most importantly, the major axis of the ellipse is aligned along the diagonal $y=x$ in the plot, which means that there is a higher probability of finding trabeculae with similar thickness next to each other, i.e., thin ones next to thin ones rather than next to thick ones.

\section{Discussion}

In this work, we studied bone remodeling of trabecular bone based on a dynamic cellular structure where mechanoregulated remodeling events result in a thinning or thickening of trabeculae. The aim was to use an idealized description of trabecular bone to understand its architectural stability during ongoing remodeling and not to predict age-related changes like trabecular bone loss or the transition from a more plate-like to a more rod-like architecture (Stauber and Müller 2006). Consequently, we aimed at a compromise in the model formulation with a simplified description of architecture and loading, while maintaining the main characteristics of mechano-regulated trabecular bone remodeling, in particular with a careful parameter choice. In the skeleton, the loading scenario and the corresponding trabecular arrangement are much more complex than a cubic lattice under hydrostatic compression. Different skeletal sites show distinct trabecular patterns and the basic organization of the trabeculae along the vertical and the horizontal direction is a reasonable approximation for the vertebral body only; it is surely not appropriate to describe the trabecular network in other well-studied skeletal locations such as the femoral head or the distal radius. Previous works have shown the ability of simplified microstructural models representing the trabecular network as a cubic lattice to capture main mechanical features of vertebral bone, like the density-elasticity relationship or the mechanical consequences of trabecular thinning and loss (Jensen et al. 1990; Yeh and Keaveny 1999; Gibson 2005). Shortcomings of this idealized depiction are obvious since it is known, for instance, that vertebral trabecular architecture shows a gradient from a finer to a more coarse structure when moving from the endplates to the center (Smit et al. 1997). Trabecular bone, especially in young individuals, also has plate-like elements (Liu et al. 2008) which are not considered in our model. Furthermore, in a real vertebra the loading is higher along the vertical direction compared to perpendicular directions (Adams and Dolan 1995). This fact produces an anisotropic distribution of bone material with obviously more bone oriented vertically (Thomsen et al. 2013). However, in agreement with other in silico investigations of trabecular bone remodeling (Huiskes et al. 2000; Ruimerman et al. 2005), we applied the same force along vertical and horizontal directions to avoid structural anisotropy (caused by anisotropic loading) and, consequently, an analysis which has to separate between vertical and horizontal trabeculae (Thomsen et al. 2013). In our model, a higher vertical load would simply result in thicker vertical trabeculae. This would complicate the interpretation of our results as, for instance, the correlation between the thicknesses of neighboring trabeculae (Fig. 6) since a thicker trabecula could now have two explanations: $(i)$ the architectural environment of the trabecula or (ii) the higher load due to its better vertical 
alignment. In conclusion, using a more realistic description of bone architecture and loading conditions not only results in a strong increase of the computational effort, but even more important, the interpretation of the simulation results with respect to the research question becomes problematic. Nevertheless, the limitations of our approach deserve further attention in the future.

In this work, we described the local mechanical environment using strain energy density (SED) and, in principle, different measures of the mechanical environment may lead to different responses in mechano-regulated remodeling simulations. This is most evident when the material properties of the virtual tissue are strongly heterogeneous (e.g., during bone healing), and therefore, the relation between various local mechanical quantities like stress, strain and SED is governed by the spatially varying elastic properties of the tissue (Vetter et al. 2012). However, in our model, bone is described as a homogeneous material, i.e., we assigned to each trabecula the same elastic constants. Under this condition, SED is directly proportional to the second power of stress or strain. Being the range of SED quite limited, as indicated by the sharp peak-shaped SED distribution (Fig. 2d) where roughly $80 \%$ of the observed SED values are within two peak widths (measured at half maximum), the effect of the nonlinearity (power operator) is expected to be negligible. Regions of high/low SED match with regions of high/low stress(strain) and our results should therefore be rather independent of the quantity used to measure the local mechanical environment. For our work, we chose the SED as mechanical stimulus since it has been shown that SED correlates well with adaptive bone formation and bone resorption in living animals (Schulte et al. 2013a) as well as in humans (Christen et al. 2014).

Generally, the evolution in time drives the virtual bone toward a steady-state configuration with only minimal changes after 40 years. In this configuration, all the trabeculae have a similar SED, where local disorder in the cubic network architecture is compensated by a variability in the thickness of the trabeculae. We observed an interesting exception of this behavior when the system is hardly mechano-responsive, i.e., for high values of the remodeling parameter $S E D_{\text {ref }}$, in a regular cubic lattice. In this case (Fig. 2d), the SED distribution as well as the trabecular thickness distribution became bimodal in shape. Although the trabecular thickness distribution does not change in time, a closer investigation showed that the thickness of the individual trabeculae fluctuates. The regular lattice is a special case since the trabeculae are loaded only axially, and consequently, the SED in a trabecula can be easily calculated to be proportional to the negative fourth power of the thickness of the trabecula (Eq. B1, Appendix B). After several iterations resulting in a rough homogenization of the thicknesses of the trabeculae, the force on a single trabecula hardly depends on the other trabeculae. Therefore, a recurrence relation for the time evolution of the trabecular cross- sectional area can be written down (Eq. B3, Appendix B) and analyzed using tools of nonlinear dynamics (Strogatz 2001). The analysis shows that the system becomes unstable when the mechano-sensitivity is reduced with $\mathrm{SED}_{\text {ref }}$ larger than $7.4 \times 10^{6} \mathrm{~J} / \mathrm{m}^{3}$ (Eq. B7, Appendix B). We assume that such a "pathological" behavior cannot be observed in real bone and is largely a result of model properties, in particular the fact that remodeling events occur instantaneously and at the same time at different sites in the virtual bone.

Another important model assumption is that some architectural disorder in form of a deviation from a completely regular cubic lattice is "frozen" into the system, i.e., is unaltered by remodeling. Consequently, our model does not allow an architectural adaptation as observed in real bone (Huiskes 2000; Fratzl and Weinkamer 2007). We deliberately chose a model description with fixed architectural disorder, even though incorporating adaptation is straightforward. Using, for example, a Monte Carlo approach the joints, i.e., the points in the lattice where six trabeculae meet, could be potentially moved. Exploiting the standard Metropolis rule used in Statistical Physics (Binder 2010), a decision is then made whether the joint is moved depending on the associated change in SED. However, in this scenario with the system under hydrostatic compression, the steady-state configuration is easily predictable: a fully ordered regular lattice minimizing SED. Two factors in real bone contribute that some architectural disordered remains in the trabecular network. Firstly, probably not all of remodeling is mechanically controlled, but also the so-called untargeted remodeling (Burr 2002; Parfitt 2002) takes place serving other functions beyond mechanical needs like mineral homeostasis. Secondly, although the main loading on a vertebra is along the spine, the loading necessarily shows some variability both in directionality and magnitude. In our model, in which remodeling occurs mechanically controlled in a deterministic way and the external loading is fixed, the essential architectural disorder was therefore implemented into the lattice by a predefined unalterable degree.

Concerning the results of our study, a first important outcome is that the system-starting from an artificial initial architecture-approaches a steady state after about 30 40 years in all cases with only the "pathological" exceptions discussed above. The steady state is characterized by a uniform SED in all trabeculae. The initial increase in $B V / T V$ (Figs. 2b, 3a) can be understood by the fact that in the initial configuration the trabecular thickness is Gaussian distributed, which corresponds to a strongly asymmetric initial distribution of the SED (Appendix A and Fig. 2d). Trabeculae are more likely to have a SED above SED ref $_{\text {, and con- }}$ sequently, deposition prevails in this early phase of about 8 years. A second result concerns the different influences of architectural disorder. The initial overshooting of $B V / T V$ is largest for largest disorder (Fig. 2b), and some part of this 
increase in $B V / T V$ is conserved until the steady state. An opposing trend is observed for the loss of trabeculae with disorder (Fig. 4): More trabeculae are lost for higher disorder. The architectural disorder together with the loss of trabeculae weakens the virtual bone mechanically, therefore deforming more and receiving a larger mechanical stimulation. Remodeling then leads to deposition on mechanically stimulated trabeculae and an overall increase in bone volume, thereby overcompensating the loss of trabeculae. It is also worth mentioning that in lattices with larger disorder the "pathological" behavior reflected in bimodal trabecular thickness distributions (see above) were less evident. Architectural disorder has a stabilizing effect on the dynamic behavior of the virtual bone.

Probably, most relevant for understanding the mechanobiology of trabecular bone are the results obtained concerning the spatial distribution of the bone volume. The often told simplified story about the "saving quality" of mechanics for bone architecture is questionable. Only for a single isolated trabecula does a thinning of a trabecula increase its mechanical stimulation, and the triggering of deposition "saves" it from resorption. However, for the case of the trabecula being part of a network with architectural disorder (like in real bone), there is no correspondence between the thickness of the trabecula and the received stimulation. Remodeling not only leads to a loss of trabeculae (Fig. 4), but the neighborhood of lost trabeculae is characterized by thinner trabeculae than average (Fig. 5), resulting in a further decrease of the local bone volume. On this local length scale of a trabecula and its nearest neighborhood, mechanically regulated remodeling results in structural heterogeneity rather than in a homogenization of the bone volume. Our simulations therefore predict a heterogeneity of bone volume on a length scale of about one millimeter as a result of bone remodeling.

The implication of our results for bone quality suggests that, although mechanical stimulation has a beneficial effect for preserving bone mass (and hence stiffness), as postulated already by Frost (Frost 1987) in the framework of his mechano-stat theory and demonstrated in several mechanobiological experiments (Robling et al. 2006; Gerhard et al. 2009; Lambers et al. 2013), the ability of mechanical forces to preserve bone architecture is questionable. Indeed, increasing mechanical load causes an increase in bone mass mainly by thickening some trabeculae rather than by increasing (or at least preserving) the total number of trabeculae in the network (Lambers et al. 2011, 2013; Saparin et al. 2011). Hence, the task to design physical exercises, which are particularly beneficial for bone architectural quality, seems hard to be addressed only by in vivo experiments. Computational efforts, also with larger and more sophisticated models than the one employed in this study (Levchuk et al. 2014), can play a supportive role, especially in preselecting physical therapies that are subsequently tested in animal experiments.

\section{Appendix A}

Relationship between trabecular thickness and strain energy density frequency distributions

With $T$ the thickness of a single trabecula having a Young's modulus $E$ and subjected to an axial load $F$, the strain energy density SED can be written as:

$\operatorname{SED}(T)=g(T)=\frac{8}{\pi^{2}} \frac{F^{2}}{E}\left(\frac{1}{T}\right)^{4}$.

Considering a collection of independent trabeculae and assuming that $T$ has a Gaussian probability density function $n_{T}(T)$ (blue curve, Fig. 2c) with mean value $\mu$ and standard deviation $\sigma$ :

$n_{T}(T)=\frac{1}{\sigma \sqrt{2 \pi}} \exp \left(-\frac{(T-\mu)^{2}}{2 \sigma^{2}}\right)$,

the probability density function of SED is calculated as (blue curve, Fig. 2d):

$$
\begin{aligned}
& f_{S}(\mathrm{SED})=n_{T}\left(g^{-1}(\mathrm{SED})\right)\left|\frac{d g^{-1}(\mathrm{SED})}{d(\mathrm{SED})}\right| \\
& =\frac{\gamma^{1 / 4}}{4 \sigma \sqrt{2 \pi}}\left(\frac{1}{\mathrm{SED}}\right)^{5 / 4} \exp \left\{-\frac{\left[\left(\frac{\gamma}{\mathrm{SED}}\right)^{1 / 4}-\mu\right]^{2}}{2 \sigma^{2}}\right\},
\end{aligned}
$$

where $\gamma=\frac{8 F^{2}}{\pi^{2} E}$.

\section{Appendix B}

Mechanical control of the remodeling of a single trabecula: recurrence relation

Considering one single trabecula loaded by a constant axial force $F$ and characterized by a Young's modulus $E$, the relationship between the cross-sectional area $A$ and the strain energy density SED at a discrete time point $i$ is:

$\operatorname{SED}_{i}=\frac{F^{2}}{2 E}\left(\frac{1}{A_{i}}\right)^{2}$.

According to the remodeling rule introduced in Fig. 2a and assuming linearity (i.e., $\mathrm{SED}$ remains smaller than $2 \mathrm{SED}_{\text {ref }}$ ), the change in cross-sectional area $\Delta A$ is given by:

$\Delta A=\Delta A_{\max }\left(\frac{\mathrm{SED}}{\operatorname{SED}_{\text {ref }}}-1\right)$.

Hence, by inserting (B1) into (B2), the recurrence relation for the cross-sectional area reads: 


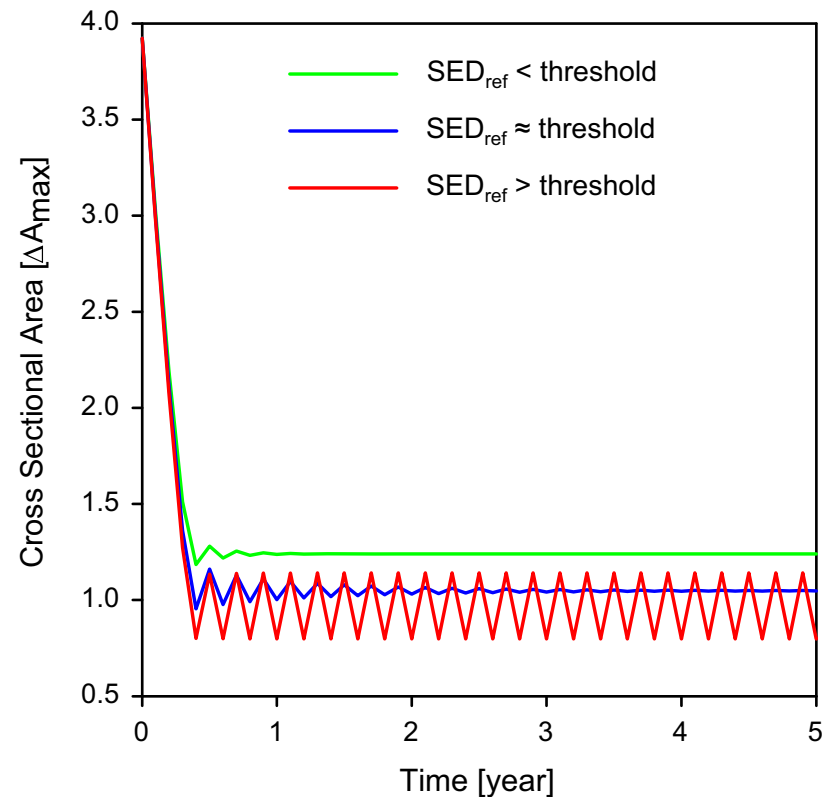

Fig. 7 Time evolution of the cross-sectional area of an individual trabecula having an initial thickness of $0.14 \mathrm{~mm}$ and remodeled according to the recurrence relation derived in Appendix B (Eq. (B7)) for three different values of $\mathrm{SED}_{\text {ref }}$. When $\mathrm{SED}_{\text {ref }}$ is well below the instability threshold (e.g., $\mathrm{SED}_{\text {ref }}=5 \times 10^{6} \mathrm{~J} / \mathrm{m}^{3}<7.4 \times 10^{6} \mathrm{~J} / \mathrm{m}^{3}$ ), the trabecula can be controlled by a mechano-regulated remodeling process. If $\mathrm{SED}_{\text {ref }}$ approaches the instability threshold (e.g., $\operatorname{SED}_{\text {ref }}=7 \times 10^{6} \mathrm{~J} / \mathrm{m}^{3}$ ), despite some initial oscillations, the cross-sectional area still converges to a steady state. For values of $\mathrm{SED}_{\text {ref }}$ above the instability threshold (e.g., $\mathrm{SED}_{\text {ref }}=9 \times 10^{6} \mathrm{~J} / \mathrm{m}^{3}$ ), the cross-sectional area oscillates without approaching a steady state

$A_{i+1}=A_{i}+\Delta A=A_{i}+\Delta A_{\max }\left(\frac{F^{2}}{2 E \mathrm{SED}_{\text {ref }}} \frac{1}{A_{i}^{2}}-1\right)$.

The additional normalization by the factor $\Delta A_{\max }$ (for its definition see Method section) allows writing (B3) in a dimensionless form:

$x_{i+1}=x_{i}+\hat{\gamma} \frac{1}{x_{i}^{2}}-1$,

with $x_{i}=\frac{A_{i}}{\Delta A_{\max }}$ and $\hat{\gamma}=\frac{F^{2}}{2 E \operatorname{SED}_{\operatorname{ref}}} \frac{1}{\Delta A_{\max }^{2}}$. The fixed points of a general recurrence relation $x_{i+1}=f\left(x_{i}\right)$ are found by setting $f\left(x^{*}\right)=x^{*}($ Strogatz 2001), hence:

$x^{*}=x^{*}+\hat{\gamma} \frac{1}{x^{* 2}}-1$

resulting in

$x^{*}= \pm \sqrt{\hat{\gamma}}$.

The stability of the fix points (B6) can be then analyzed by looking at the first derivative of the recurrence relation (B4):

$\lambda=f^{\prime}\left(x^{*}\right)=1-\frac{2}{\sqrt{\hat{\gamma}}}$.
The model is unstable when $|\lambda|>1$, hence for $0<\hat{\gamma}<1$. Assuming $F=1.5 \mathrm{~N}, E=10 \mathrm{GPa}$ and $\Delta A_{\max }=0.0039 \mathrm{~mm}^{2}$, the corresponding value of $\mathrm{SED}_{\text {ref }}$ above which the recurrence relation gives rise to oscillations in the cross-sectional area is $7.4 \times 10^{6} \mathrm{~J} / \mathrm{m}^{3}$ (Fig. 7).

\section{References}

Adams MA, Dolan P (1995) Recent advances in lumbar spinal mechanics and their clinical-significance. Clin Biomech 10(1):3-19

Binder K a H D (2010) Monte Carlo simulation in statistical physics: an introduction. Springer, New York

Burr DB (2002) Targeted and nontargeted remodeling. Bone 30(1):2-4

Carretta R, Luisier B, Bernoulli D, Stussi E, Müller R, Lorenzetti S (2013) Novel method to analyze post-yield mechanical properties at trabecular bone tissue level. J Mech Behav Biomed Mater 20:618

Chavassieux PM, Arlot ME, Reda C, Wei L, Yates AJ, Meunier PJ (1997) Histomorphometric assessment of the long-term effects of alendronate on bone quality and remodeling in patients with osteoporosis. J Clin Invest 100(6): 1475-1480

Christen P, Ito K, Ellouz R, Boutroy S, Sornay-Rendu E, Chapurlat RD, van Rietbergen B (2014) Bone remodelling in humans is loaddriven but not lazy. Nat Commun 5. doi:10.1038/ncomms5855

Dunlop J, Hartmann M, Bréchet Y, Fratzl P, Weinkamer R (2009) New suggestions for the mechanical control of bone remodeling. Calcified Tissue Int 85(1):45-54

Easley SK, Chang MT, Shindich D, Hernandez CJ, Keaveny TM (2012) Biomechanical effects of simulated resorption cavities in cancellous bone across a wide range of bone volume fractions. J Bone Miner Res 27(9):1927-1935

Fratzl P, Weinkamer R (2007) Nature's hierarchical materials. Prog Mater Sci 52(8):1263-1334

Frost HM (1987) Bone mass and the mechanostat-a proposal. Anat Rec 219(1):1-9

Gerhard FA, Webster DJ, van Lenthe GH, Müller R (2009) In silico biology of bone modelling and remodelling: adaptation. Philos T R Soc A 367(1895):2011-2030

Gibson LJ (2005) Biomechanics of cellular solids. J Biomech 38(3):377-399

Guo XE, Kim CH (2002) Mechanical consequence of trabecular bone loss and its treatment: a three-dimensional model simulation. Bone 30(2):404-411

Hartmann MA, Dunlop JWC, Bréchet YJM, Fratzl P, Weinkamer R (2011) Trabecular bone remodelling simulated by a stochastic exchange of discrete bone packets from the surface. J Mech Behav Biomed Mater 4(6):879-887

Hernandez CJ, Keaveny TM (2006) A biomechanical perspective on bone quality. Bone 39(6):1173-1181

Hildebrand T, Laib A, Müller R, Dequeker J, Ruegsegger P (1999) Direct three-dimensional morphometric analysis of human cancellous bone: microstructural data from spine, femur, iliac crest, and calcaneus. J Bone Miner Res 14(7):1167-1174

Huiskes R (2000) If bone is the answer, then what is the question? J Anat 197:145-156

Huiskes R, Ruimerman R, van Lenthe GH, Janssen JD (2000) Effects of mechanical forces on maintenance and adaptation of form in trabecular bone. Nature 405(6787):704-706

Jee WSS (2001) Bone mechanics handbook. CRC Press, Boca Raton

Jensen KS, Mosekilde L, Mosekilde L (1990) A model of vertebral trabecular bone architecture and its mechanical-properties. Bone 11(6):417-423 
Kabel J, Odgaard A, van Rietbergen B, Huiskes R (1999) Connectivity and the elastic properties of cancellous bone. Bone 24(2):115-120

Keaveny TM, Morgan EF, Niebur GL, Yeh OC (2001) Biomechanics of trabecular bone. Annu Rev Biomed Eng 3:307-333

Lambers FM, Koch K, Kuhn G, Ruffoni D, Weigt C, Schulte FA, Müller R (2013) Trabecular bone adapts to long-term cyclic loading by increasing stiffness and normalization of dynamic morphometric rates. Bone 55(2):325-334

Lambers FM, Schulte FA, Kuhn G, Webster DJ, Müller R (2011) Mouse tail vertebrae adapt to cyclic mechanical loading by increasing bone formation rate and decreasing bone resorption rate as shown by time-lapsed in vivo imaging of dynamic bone morphometry. Bone 49(6): 1340-1350

Levchuk A, Zwahlen A, Weigt C, Lambers FM, Badilatti SD, Schulte FA, Kuhn G, Müller R (2014) The clinical biomechanics award 2012 - presented by the European society of biomechanics: large scale simulations of trabecular bone adaptation to loading and treatment. Clin Biomech 29(4):355-362

Liu XS, Bevill G, Keaveny TM, Sajda P, Guo XE (2009) Micromechanical analyses of vertebral trabecular bone based on individual trabeculae segmentation of plates and rods. J Biomech 42(3):249 256

Liu XS, Sajda P, Saha PK, Wehrli FW, Bevill G, Keaveny TM, Guo XE (2008) Complete volumetric decomposition of individual trabecular plates and rods and its morphological correlations with anisotropic elastic moduli in human trabecular bone. J Bone Miner Res 23(2):223-235

Luxner MH, Stampfl J, Pettermann HE (2005) Finite element modeling concepts and linear analyses of 3D regular open cell structures. J Mater Sci 40(22):5859-5866

Luxner MH, Stampfl J, Pettermann HE (2007) Numerical simulations of 3D open cell structures - influence of structural irregularities on elasto-plasticity and deformation localization. Int J Solids Struct 44(9):2990-3003

Luxner MH, Stampfl J, Pettermann HE (2009a) Nonlinear simulations on the interaction of disorder and defects in open cell structures. Comp Mater Sci 47(2):418-428

Luxner MH, Woesz A, Stampfl J, Fratzl P, Pettermann HE (2009b) A finite element study on the effects of disorder in cellular structures. Acta Biomater 5(1):381-390

Mulvihill BM, McNamara LM, Prendergast PJ (2008) Loss of trabeculae by mechano-biological means may explain rapid bone loss in osteoporosis. J R Soc Interface 5(27):1243-1253

Nazarian A, Stauber M, Zurakowski D, Snyder BD, Müller R (2006) The interaction of microstructure and volume fraction in predicting failure in cancellous bone. Bone 39(6):1196-1202

Parfitt AM (1994) Osteonal and hemi-osteonal remodeling - the spatial and temporal framework for signal traffic in adult human bone. $\mathrm{J}$ Cell Biochem 55(3):273-286

Parfitt AM (2002) Targeted and nontargeted bone remodeling: relationship to basic multicellular unit origination and progression. Bone 30(1):5-7

Parfitt AM, Drezner MK, Glorieux FH, Kanis JA, Malluche H, Meunier PJ, Ott SM, Recker RR (1987) Bone histomorphometrystandardization of nomenclature, symbols, and units. J Bone Miner Res 2(6):595-610

Robling AG, Castillo AB, Turner CH (2006) Biomechanical and molecular regulation of bone remodeling. Annu Rev Biomed Eng 8:455498

Ruffoni D, Dunlop JWC, Fratzl P, Weinkamer R (2010) Effect of minimal defects in periodic cellular solids. Philos Mag 90(13):1807_ 1818
Ruffoni D, Müller R, van Lenthe GH (2012a) Mechanisms of reduced implant stability in osteoporotic bone. Biomech Model Mechan 11(3-4):313-323

Ruffoni D, Wirth AJ, Steiner JA, Parkinson IH, Müller R, van Lenthe GH (2012b) The different contributions of cortical and trabecular bone to implant anchorage in a human vertebra. Bone 50(3):733738

Ruimerman R, Hilbers P, van Rietbergen B, Huiskes R (2005) A theoretical framework for strain-related trabecular bone maintenance and adaptation. J Biomech 38(4):931-941

Rusconi M, Valleriani A, Dunlop JWC, Kurths J, Weinkamer R (2012) Quantitative approach to the stochastics of bone remodeling. Epl Europhys Lett 97(2):28009

Saparin P, Scherf H, Hublin JJ, Fratzl P, Weinkamer R (2011) Structural adaptation of trabecular bone revealed by position resolved analysis of proximal femora of different primates. Anat Rec 294(1):5567

Schulte FA, Ruffoni D, Lambers FM, Christen D, Webster DJ, Kuhn G, Muller R (2013a) Local mechanical stimuli regulate bone formation and resorption in mice at the tissue level. PLoS ONE $8(4): \mathrm{e} 62172$

Schulte FA, Zwahlen A, Lambers FM, Kuhn G, Ruffoni D, Betts D, Webster DJ, Mueller R (2013b) Strain-adaptive in silico modeling of bone adaptation - a computer simulation validated by in vivo micro-computed tomography data. Bone 52(1):485-492

Seeman E, Delmas PD (2006) Mechanisms of disease — bone qualitythe material and structural basis of bone strength and fragility. New Engl J Med 354(21):2250-2261

Smit TH, Burger EH (2000) Is BMU-coupling a strain-regulated phenomenon? A finite element analysis. J Bone Miner Res 15(2):301307

Smit TH, Odgaard A, Schneider E (1997) Structure and function of vertebral trabecular bone. Spine 22(24):2823-2833

Stauber M, Müller R (2006) Age-related changes in trabecular bone microstructures: global and local morphometry. Osteoporosis Int 17(4):616-626

Stauber M, Rapillard L, van Lenthe GH, Zysset P, Müller R (2006) Importance of individual rods and plates in the assessment of bone quality and their contribution to bone stiffness. J Bone Miner Res 21(4):586-595

Strogatz SH (2001) Nonlinear dynamics and chaos. Westview Press, Boulder

Thomsen JS, Niklassen AS, Ebbesen EN, Bruel A (2013) Age-related changes of vertical and horizontal lumbar vertebral trabecular $3 \mathrm{D}$ bone microstructure is different in women and men. Bone 57(1):47-55

Tsubota K, Adachi T, Tomita Y (2002) Functional adaptation of cancellous bone in human proximal femur predicted by trabecular surface remodeling simulation toward uniform stress state. J Biomech 35(12):1541-1551

van Lenthe GH, Stauber M, Müller R (2006) Specimen-specific beam models for fast and accurate prediction of human trabecular bone mechanical properties. Bone 39(6):1182-1189

Vetter A, Witt F, Sander O, Duda GN, Weinkamer R (2012) The spatiotemporal arrangement of different tissues during bone healing as a result of simple mechanobiological rules. Biomech Model Mechanobiol 11(1-2):147-160

Yeh OC, Keaveny TM (1999) Biomechanical effects of intraspecimen variations in trabecular architecture: a three-dimensional finite element study. Bone 25(2):223-228 